\title{
Electroacupuncture System using Fuzzy Rules
}

\author{
[ Yu-Sik Hong, Ji-Ho Cho, Geuk Lee*]
}

\begin{abstract}
Recently, the scientific study from traditional oriental medicine is being progressed. Moreover, through both oriental medicine and western medicine are cooperated with medical examination and treatment, the study in cooperation of merits from oriental medicine as well as from western medicine is progressed. In this paper, we will develop automatic needle shot using fuzzy rules and USN techniques, which a user can choose meridian pathways from the menu items built in the cellular phone, so it can perceive patient's body conditions and launch an aneurysm electric pressure of a cycle.
\end{abstract}

Keywords—Pulse wave, Fuzzy rules, Expert system

\section{Introduction}

Many western style hospitals are efficiently established in EMR for patients treatments and managements but most traditional oriental hospitals are not using EMR charts. Traditional oriental hospitals are using pulse, auscultation, tongue, paperweight for treating patients diseases. The concept of tongue in oriental care is observing the color and state of tongue and decides the state of patients health. And the concept of pulse is observing the state of fast or slow pulse, the strength of pulse and the depth of pulse, so decides the one type among various 28 types for the diseases of patients. If the patient has the strong wave of pulse and the good data of tongue, it is decided as a good conditions. But if the patient has the weak wave of pulse, it is decided to do more precise tests. Because in traditional oriental care, the pulse data of patients can be changed from the state of patients depending on the sex, ages, the thickness of blood vessel. In this paper, to solve these problems considering the body pulse and tongue state and using the fuzzy logic and fuzzy inference rule, the precise deciding procedure of patients disease are simulated. In this paper, using the fuzzy logic and fuzzy inference rule, the procedures of appropriate optimum electroacupucture estimation time of patients body conditions are simulated. In this paper, to estimate precise and appropriate optimum electroacupucture time, it is divided into 3 conditions as body conditions, disease conditions and age condition. But because these classification techniques are varied from the disease condition of patients, in this paper, to improve these problems the precise and appropriate optimum electroacupucture time is estimated for considering disease conditions and using fuzzy rules.

\section{You-Sik Hong}

School of computer science Information Engineering, Sangii University Won-ju, Republic of Korea

Ji-Ho Cho / Geuk Lee*

Department of Computer Engineering, Hannam University

Dae-Jeon, Republic of Korea

*Geuk Lee is Corresponding Author
Usual electroacupucture time is fixed as 30 minutes, but in this paper for considering the conditions of patients, the electroacupucture times are simulated as adjusting the elongated times or shortened times. Because usual electroacupucture did not know the correct electroacupucture point, therefore many difficulties are existed. But in this paper if we input the disease name through the mobile phone on the initializing screen, we can see the correct point on the mobile phone screen, so these difficult problems are solved and improved. This paper are constructed as follows. In chapter 2, electroacupucture algorithm is shown. In chapter 3 , the body pulse and acupucture points for making an artificial electroacupucture is shown and in chapter 5, the results of electroacupucture simulation is shown and in chapter 5 is shown the conclusions.

\section{Electroacupuncture Algorithm}

In this paper, as shown in "Fig. 1", when the electrical stimulation is applied to the PAD of each finger, accordingly to the painful position, the algorithm, which senses the varying states of small signal voltage of body and decides the name of disease, is proposed. Originally if the human hurts the body, the position has a higher resistance than the other positions of the electrical resistance. Because originally human has a unique current value, the wound position has the higher electrical resistance, so it has the characteristics that the currents cannot pass well. Therefore the wound position passes small currents, so it has a characteristics that the absolute current values of cell become small. Using multi PAD and knowledge of oriental care, when electrical shock is applied to 5 fingers, if feels pains, the reflection area of the finger has problems, so it is simulated for predicting the disease of patients. Therefore when the electrical shock of acupuncture blood points is applied to liver based pulse, which is positioned on the hands of reflection area, if the pain happens in the main refection area of the surrounding liver, the doctor decides the disease problems of liver based pulse and so, in this paper, the hardware system is made for deciding the disease of patients using multi PAD.

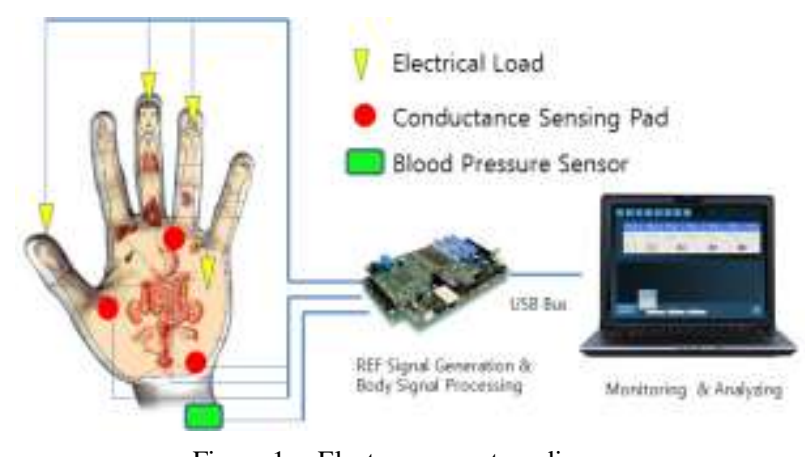

Figure 1. Electroacupuncture diagram. 
The first parts are composed of sensor, which senses pulses diagnosis and electrical conductance of body position of problems, and sensing parts, which depending on the state of patients, generating the reference signal and controlling the weak or strong electrical pattern. The second hardware part is DSP(Digital Signal Processor) Board, which generates signals and estimates and divides the signals using fuzzy recognition.

The last third part is shown the real time sended signal on the screen on DSP board and is consisted of the analyzing software, which helps the decision for the patient's state, and computer hardware. In this paper, DSP Board experiments for artificial intelligence body pulse decision system is designed for appropriate and various patterns and also according to the painful area, sensing the varying state of small body signal voltage is designed. "Fig. 1" shows the results of DSP simulation for blood points of electroacupuncture. Generally in human body, if occurs various diseases, cancers and infection reactions, human shows pains, an affected parts, heats, edemas and seizures. Therefore if human has pains, an affected parts on the skin and body organization, body and muscles shrink for protecting oneself. If an affected parts on the skin and body organization or muscles shrink, the mounts of bloods are diminished and so the amounts of oxygen's are also diminished and the amounts of nutrition's are diminished. Also the bodily wastes don't excrete and can be piled up.

\section{Disease Decision Algorithm}

In this paper, it tried to solve these problems using intelligent fuzzy rules.

$$
\begin{aligned}
& \mathrm{e}=\mathrm{R}-\mathrm{Y} \\
& \mathrm{Ce}=\mathrm{e} 2-\mathrm{e} 1
\end{aligned}
$$

\section{Where, Y: optimum pulse feeling judgment \\ R: Criteria Input \\ e: Error \\ Ce: Error Displacement \\ e2: Current Error}

A global priority reflects the importance of an element with respect to the focus of the problem. The derivation of local priorities is carried out through the use of a comparison scale and a pairwise comparison matrix. A comparison matrix for deriving the priority vector.

$$
w^{T}=\left(w_{1}, w_{2}, w_{3}, w_{4}, \cdots\right) \text { is associated }
$$

with 3 elements in a specific level with respect to a single element in a level immediately about it. Such a matrix is denoted by A.

$$
A=\left[\begin{array}{lllllllll}
w_{1} / w_{1} & w_{1} / w_{2} & w_{1} / w_{3} & w_{1} / w_{4} \\
w_{2} / w_{1} & w_{2} / w_{2} & w_{2} / w_{3} & w_{2} / w_{4} \\
w_{3} / w_{1} & w_{3} / w_{2} & w_{3} / w_{3} & w_{3} / w_{4} \\
w_{4} / w_{1} & w_{4} / w_{2} & w_{4} / w_{3} & w_{4} / w_{4}
\end{array}\right]
$$

In this matrix every element $a_{\text {s }}$ is the result of a pairwise comparison denoting the dominance of element $i$ relative to element $\mathrm{j}$. A comparison is also being made of the jth element with the ith element. The fuzzification membership functions in a fuzzy rule base are triangular typed ones defined by "equation (1)" with $a, b, u \in U$. The fuzzy rule base composes of MISO(Multi Input Single Output) typed rule base. Each fuzzy membership function in a fuzzy rule base has a membership value area $[0,1]$, and should be normalized in this area.

$$
y= \begin{cases}\frac{2}{b-a}(x-a), & a \leq u \leq b, \quad u \in U \\ 0, & \text { otherwise }\end{cases}
$$

This interval includes all possible values for the variable in universe of discourse $(U)$. All fuzzy sets in a fuzzy rule base have the same support interval $[a, b]$. The equation can be represented all types of fuzzy membership functions for both fuzzy and non-fuzzy membership functions. The oriental medicine is adopting 4 diagnosis methods such as seeing, hearing, inquiring, and touching but among those, there is tongue feeling system in which a doctor will see a patient's tongue for diagnosis. This used method is to observe the tongue condition or, fur deposited on it, to diagnose any disease and its kinds from the ancient times. Observation of the tongue is mainly used to understand functioning of heart and spleen. If the tongue fur is white, the corresponding disease is in its initial stage or light, indicating False Symptom, Cold Symptom, and Humid Symptom.

In this paper, to solve these problems, using artificial intelligence fuzzy technology, the appropriate electroacupun cture times and strength are controlled. Also in doing electroacupuncture, the maximum effective electroacupunc ture software, which can be done music and art therapy, is developed. In oriental care systems, by observing the state of body pulse, the health and disease states are can be seen, so the body pulse is very important to oriental care. For example if the heart does not beat and stop suddenly, this is a very dangerous state and it is a last monuments of life, so these state of heart can be decided by body pulse. From the old times, the oriental doctors considered the body pulse as a important decision data. But the problems of existing pulse decision machine are that the body pulse for detecting sensor is very difficult to find the position of exact radial artery and if the body type is different and forearm is too thick or thin, the precise pulse detection is difficult. Analog pulse detection machines have very difficult problems for quantization of pulses and if the forearm is same and one can be a thick or thin blood vessel, it is very difficult to do a precise pulse detection. Therefore to solve these problems, not only the oriental doctor decide the basic biological signal data as weak or strong and fast or slow and small or large strength of pulse simply by hands, but also the oriental doctor analyze the wave of small body pulse considering the difference of thickness of blood vessel and skin for body characteristics with basic and qualitative small and large pulse.

\section{Simulation}

The hardware configurations for artificial intelligence pulse decisions are shown in "Fig. 2". The system main DSP 
is a TMS320VC33, which is made by TI Company, and Flash ROM for operand is a HY29F040 and so it is designed for storing $512 \mathrm{~K} * 8$ Op code and has a $512 \mathrm{~K} * 32$ Main Operating RAM. In this system, A/D(Analog to Digital) Converter has 8 channel, One serial port, 1 USB Bus, 12Bit $454 \mathrm{KHz}$ Sampling Rate and D/A(Digital to Analog) Converter has 4 channel and $12 B i t 1 \mu$ s Settling Time. The estimated signal in sensor is amplified in AMP and filtered and passes Isolation Amplifier AD202. The "Fig. 2" shows the firmware flowchart of DSP Board. The execution process of firmware is divided by large 4 groups. It can be divided as a main group for main processing, a input processing group for sensor signals, a data indexing processing group for generating the exact information data based on sensed data and fuzzy logic driving group for generating the resulted data.

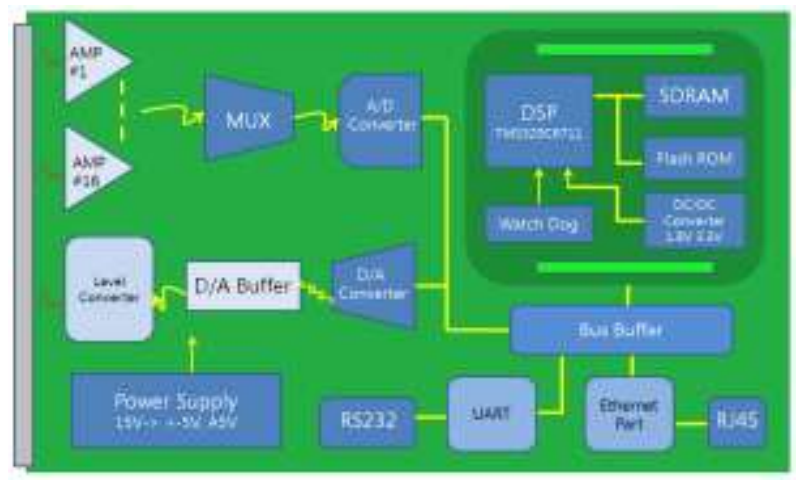

Figure 2. Block Diagram of the DSP Board for Smart Pulse Wave Decision System.

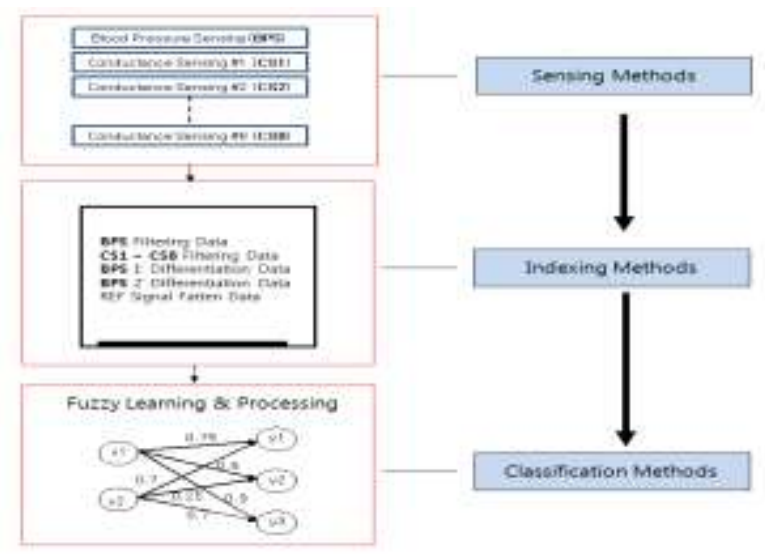

Figure 3. Intelligent Pulse wave detection system.

In "Fig. 3", using body pulse system algorithm, the dividing process is explained as 3 groups for dangerous, normal, healthy state for patients. In "Fig. 4", to decide the exact disease for patients, by second differential equating and by deciding the aging state for patient's blood, the decision process for patient's disease prediction is explained.

In this paper, when the same disease name of body pulse data has two more different decision values, to do an exact decision, the used trust value combination function is used to calculate the trust values again.

$$
\begin{aligned}
& \beta \mathrm{c}=\beta \operatorname{comb}(\beta \mathrm{c}, \beta \text { cold }) \\
& =\max (\beta \mathrm{c}, \beta \text { cold })
\end{aligned}
$$

Here the $\beta$ cold is the resulting trust value for path of decision and $\beta \mathrm{c}$ is another resulting trust value for other path of decision. Generally fuzzy rules are expressed as

IF-THEN instructions and fuzzy inference is a procedure for interfering the new relations or facts from the given rules.

$$
\begin{aligned}
& \text { Input : } \quad \mathrm{x} \text { is } \mathrm{A}^{\prime} \text { AND } \mathrm{y} \text { is } \mathrm{B}^{\prime} \\
& \mathrm{R} 1 \text { : IF } \mathrm{x} \text { is } \mathrm{A} 1 \mathrm{AND} \mathrm{y} \text { is } \mathrm{B} 1 \text {, THEN } \mathrm{z} \text { is } \mathrm{C} 1 \\
& \text { OR R2 : IF } \mathrm{x} \text { is A2 AND } \mathrm{y} \text { is } \mathrm{B} 2 \text {, THEN } \mathrm{z} \text { is } \mathrm{C} 2 \\
& \text { OR Rn : IF } \mathrm{x} \text { is An AND y is Bn, THEN } \mathrm{z} \text { is } \mathrm{Cn} \\
& \text { Conclusion : } \mathrm{z} \text { is } \mathrm{C} \text { joint function }
\end{aligned}
$$

: Fuzzy decision rule has more than two different trust values. For these cases, the function, which recalculates the trust values of decision, is joint function for trust values. For example, if some patient has a probability for aortic insufficiency is 0.3 , the normal probability is 0.7 . But in ambiguous conditions, if some patient has a probability for aortic insufficiency is 0.3 , fuzzy measurement cannot decide normal condition as 0.7 for aortic valve. Because physical condition, health condition and other diseases for patients have many variables, the normal probability for aortic valve may be 0.5 or 0.9 . In table 2 , if the disease has same disease, the fuzzy rule is explained as considering for weight, age, sex conditions. The function for automatic search for tongue color checkup is shown for same patients. Therefore in this paper, to do the more exact prediction for patient, it is proposed to measure for automatic search for tongue and body pulse checkup and proposed the algorithm and proposed the simulation for exact decision. .But to measure simultaneously for automatic search for tongue and body pulse checkup, the patient's conditions for body, age, sex are considered and the algorithm for deciding trust values about disease confidence level is proposed.

$$
\begin{aligned}
& \text { IF E1 AND E2 AND E3 THEN H } \\
& \mathrm{CF}(\mathrm{H}, \mathrm{E})=\mathrm{CF}(\mathrm{H}, \mathrm{E} 1 \wedge \mathrm{E} 2 \wedge \mathrm{E} 3)=0.8 \\
& \mathrm{MB}(\mathrm{E} 1, \mathrm{e})=0.6, \mathrm{MD}(\mathrm{E} 1, \mathrm{e})=0, \mathrm{CF}(\mathrm{E} 1, \mathrm{e})=0.6 \\
& \mathrm{MB}(\mathrm{E} 2, \mathrm{e})=0.3, \mathrm{MD}(\mathrm{E} 2, \mathrm{e})=0, \mathrm{CF}(\mathrm{E} 2, \mathrm{e})=0.3 \\
& \mathrm{MB}(\mathrm{E} 3, \mathrm{e})=0.5, \mathrm{MD}(\mathrm{E} 3, \mathrm{e})=0, \mathrm{CF}(\mathrm{E} 3, \mathrm{e})=0.5
\end{aligned}
$$

$$
\begin{aligned}
& \mathrm{CF}(\mathrm{E}, \mathrm{e})=\mathrm{CF}(\mathrm{E} 1 \wedge \mathrm{E} 2 \wedge \mathrm{E} 3, \mathrm{e}) \\
& =\mathrm{MB}(\mathrm{E} 1 \wedge \mathrm{E} 2 \wedge \mathrm{E} 3, \mathrm{e})-\mathrm{MD}(\mathrm{E} 1 \wedge \mathrm{E} 2 \wedge \mathrm{E} 3, \mathrm{e}) \\
& =\min (\mathrm{MB}(\mathrm{E} 1, \mathrm{e}), \mathrm{MB}(\mathrm{E} 2, \mathrm{e}), \mathrm{MB}(\mathrm{E} 3, \mathrm{e}))- \\
& \quad \max (\mathrm{MD}(\mathrm{E} 1, \mathrm{e}), \mathrm{MD}(\mathrm{E} 2, \mathrm{e}), \mathrm{MD}(\mathrm{E} 3, \mathrm{e})) \\
& \quad=\min [0.5,0.6,0.3]-0 \\
& \begin{aligned}
\mathrm{CF}(\mathrm{H}, \mathrm{e})=\mathrm{CF}(\mathrm{E}, \mathrm{e}) \mathrm{CF}(\mathrm{H}, \mathrm{E}) \\
\quad=0.3 \cdot 0.7 \\
=0.21
\end{aligned}
\end{aligned}
$$

In this paper, the used learning neural network structure is as follows.

(1) Initialize the offsets and weights. 
(2) Provide to the neural networks for input and target patterns.

(3) Inverse propagate from calculating the error and data of output neural cells to the hidden levels.

$$
\begin{aligned}
e j & =t j-a j \\
\delta j & =a j(1-a j) ~ e j
\end{aligned}
$$

(4) From the inversed propagated delta, inverse propagate from calculating the error and data of hidden level neural cells.

$$
\begin{gathered}
\text { ej }=\sum \text { wjk } \delta \mathrm{k} \\
\mathrm{k} \\
\delta \mathrm{j}=\text { aj }(1-\mathrm{aj}) \mathrm{ej}
\end{gathered}
$$

(5) Control the connection weighted value by delta rules.

$$
\begin{aligned}
& \mathrm{W}(\text { new }) \mathrm{ij}=\mathrm{W}(\text { old }) \mathrm{ij}+\alpha \delta \text { iaj }+\beta \Delta \text { wij(old) } \\
& \text { bias(new)ij }=\text { bias(old)ij }+\alpha \delta .1+\beta \Delta \text { biasij(old) }
\end{aligned}
$$

(6) Repeat 1-5 processings about every input patterns.

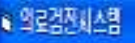

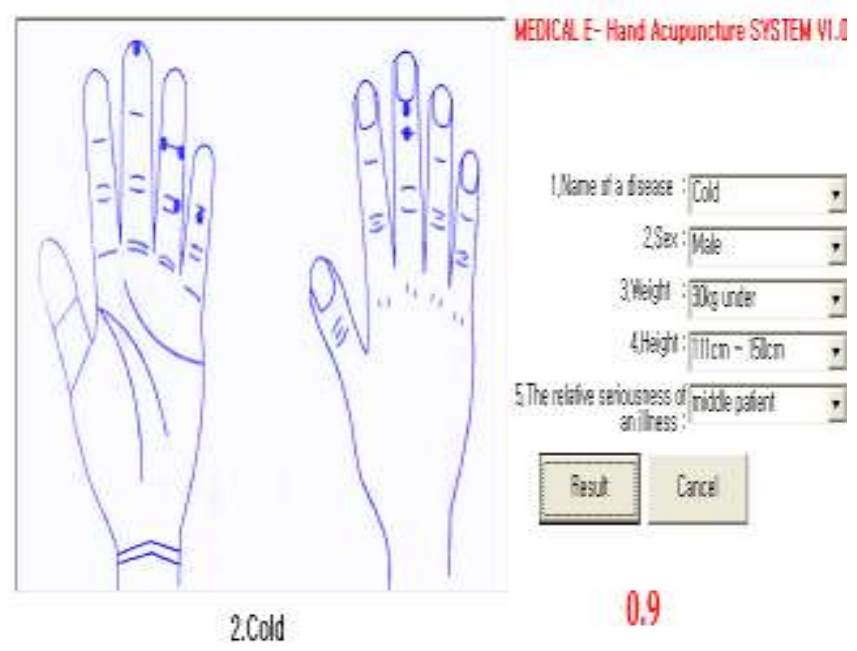

Figure 4. Electronic acupuncture needle simulation.

\section{v. Conclusions}

In this paper, using fuzzy logic and fuzzy inference rule, the estimation procedures of appropriate optimum electroacupuncture time for patient's body conditions are simulated. In this paper, the electroacupuncture that has built in pad has merits that can check automatically the patient's states and can care simultaneously according to the patient's states. To solve and improve these problems, in this paper, sensing pad, the related AMP, small signal driving circuits and DSP system products are required and the software for built In fuzzy technique that has analyzing and controlling algorithms is recommended. To solve and improve these problems, in this paper, according to the fuzzy logic and statistical and probability values, using trust values, the EMR electro chart for the name of precise diseases is simulated.

Especially for oriental care, to do a precise tests for disease, the oriental doctor must test and experiment various patient's group and must study and verify the precise trust value.

\section{Acknowledgment}

*Geuk Lee is corresponding author

\section{References}

[1] Lee Yu-Jung, Lee Jeon, Lee Hae-Jung, Ryu Hyun-Hee, Choi Eun-Ji and Kim Jong-Yeol, "Characteristic Study of the Pulse Position on CHON, KWAN and CHUCK Using the Ultrasonic Waves," Korea Journal of Orintal Medicine, vol. 13, no. 3, pp. 111-119, 2007.

[2] Lee Jeon, Lee Yujung, Lee Haejung, Choi Eunji and Kim Jongyeol, "A Case Study of Six Sigma Project for Improving method of measuring pulse wave" Korea Journal of Oriental Medicine, vol. 12, no. 2, pp. 85-92, 2006.

[3] Y. G. Lee, "Diagnostic atlas 2 analyzing pulse," Chungdam books, pp. 11-14, 2003.

[4] Serkan Gunal, and Rufat Edizkan, "Subspace based feature selection for pattern recognition," Information Sciences 2008, pp. 3716-3726.

[5] http://www.etnews.co.kr/etnews/word.

[6] Garg, M. L., Ahson, S. I., and Gupta, D. V., "A Fuzzy Petri-nets for Knowledge Represent- ation and Reasoning," Information Processing Letters, 39, pp. 165-171, 1992.

[7] Genrich, H. J., and Lautenbach, K., "System Modelling with Highlevel Petri Nets," Theo- retical Computer Science, 13, pp. 109-136, 1981.

[8] Leung, K. S., and Lam, W., "Fuzzy Concepts in Expert Systems," IEEE Computer, Sep., pp. 43-56, 1988.

[9] Looney, G. C., and Alfize, A. A., "Logical Controls via Boolean Rule Matrix Transfor-mation," IEEE Trans. on SMC, vol. 17, no. 6, Nov./Dec., pp. 1077-1082, 1987.

[10] Looney, G. C., "Fuzzy Petri Nets for Rule- based Decision Making," IEEE Trans. on SMC, vol. 18, no. 1, Jen./Feb., 1988.

[11] M. F. O'Rourke, R. P. Kelly, and A. P. Avolio, "The Arterial Pulse," 1st Ed., Lea \& Febiger, Philadelphia, 1992. 